\title{
Estudio de la conicidad de postes de madera de eucalipto (Eucalyptus globulus Labill) y pino amarillo (Pinus sp) utilizados en el Perú para la electrificación rural
}

\author{
Julio César Canchucaja Rojas*, Leonidas Miguel Castro \\ Universidad Nacional Agraria La Molina \\ Francisco García Fernández \\ Universidad Politécnica de Madrid
}

Recibido: 5 de abril del 2018 / Aprobado: 23 de mayo del 2018

doi: 10.26439/ing.ind2018.n036.2450

\begin{abstract}
Resumen: El Perú está adquiriendo postes de pino amarillo del sur y eucalipto para ejecutar proyectos de electrificación rural. A pesar de que la conicidad es una caracteristica importante para adquirir accesorios para su instalacion, no hay estudios sobre el tema. Se evaluó la conicidad de 642 postes de pino amarillo y 1590 de eucalipto, y se concluyó que existen diferencias significativas entre conicidades de ambas especies para un nivel de significación del $5 \%$.
\end{abstract}

Palabras clave: postes de madera / conicidad / electrificación rural / pinos / eucaliptos

\section{Study of the conicity of eucalyptus (Eucalyptus globulus Labill) and yellow pine (Pinus sp.) wooden poles used in Peru for rural electrification}

\begin{abstract}
Peru is purchasing southern yellow pine and eucalyptus poles to carry out rural electrification projects. Even though conicity is an important characteristic to acquire the pertinent installation accessories, there are no studies on said topic. The conicity of 642 yellow pine poles and 1,590 eucalyptus poles was evaluated, and it was concluded that there are significant differences between both species' conicities at a significance level of $5 \%$.
\end{abstract}

Keywords: wooden poles / conicity / rural electrification / pines / eucalyptus

* Correo electrónico: jccr@lamolina.edu.pe 


\section{INTRODUCCIÓN}

Actualmente en el Perú se está realizando la ampliación de la frontera eléctrica, sobre todo en las zonas rurales, con el uso mayormente de postes de madera preservados, tanto de procedencia nacional como importada, gracias a su bajo costo comparado con el de otros materiales, por su facilidad de transporte, durabilidad en servicio, entre otros. En lo que se refiere a postes de procedencia nacional, el eucalipto (Eucalyptus globulus Labill) es la especie mayormente utilizada por su abundancia, disponibilidad y bajo precio, mientras que en los importados resalta el pino amarillo del sur (Pinus sp.), de procedencia estadounidense, que completa la demanda anual de este producto, por sus propiedades físicas, mecánicas y buenas características de preservación.

En este aspecto, cuando se instalan los postes en las líneas eléctricas, se requiere de materiales de fijación para las crucetas, los conductores u otros materiales que van insertos en la zona cercana a la cabeza. Por dicho motivo, es de suma importancia conocer las circunferencias mínimas y máximas que pueden presentar las cabezas de los postes utilizados en el país, a fin de determinar las dimensiones de los elementos de sujeción arriba mencionados.

La variable que permite estimar teóricamente estas circunferencias se denomina conicidad, cuya definición indicada en la NTP 251.021 (Inacal, 2016) es "la disminución gradual del diámetro del poste del pie o base a la cabeza, expresada en $\mathrm{mm} / \mathrm{m}$ ".

La conicidad hace referencia a la disminución del diámetro del fuste desde la base hasta el ápice del árbol. Tiene su origen en la superposición del crecimiento en altura y el crecimiento en grosor que tiene el árbol y que simplificadamente le da una forma cónica (Vignote, Martínez y Villasante, 2013). Además, aunque parte de la conicidad del fuste es intrínseca a la especie y procedencia, otra parte importante es debida a la silvicultura seguida. No en vano la conicidad es una herramienta tecnológica de gran utilidad en el manejo de bosques (González, 1988).

La determinación de la conicidad de los postes utilizados en la electrificación rural es muy importante, sobre todo para determinar las dimensiones de los materiales de ferretería que se deben adquirir para colocar las crucetas u otro tipo de soporte para el sostenimiento del tendido eléctrico, telefónico, etc. 
La norma NTP 251.022 (Inacal, 2017) indica que la conicidad es característica según las especies y sus condiciones de crecimiento, la cual se calcula sobre la base de las circunferencias mínimas en la línea de tierra y en la cabeza indicadas en la tabla 3 de dicha norma (Inacal, 2017). Además, se tiene que para las longitudes y clases, que los agrupan de acuerdo con su resistencia a la carga de rotura, de postes de eucalipto las conicidades máximas permitidas fluctúan entre 10,6 y 11,3 $\mathrm{mm}$ por metro lineal.

Asimismo, en la tabla 8M de la norma ANSI 05.1 (ANSI, 2017), se tiene que para las longitudes y clases de postes de pino amarillo del sur estudiadas las conicidades máximas permitidas oscilan de 8,9 a 9,6 mm por metro lineal.

Es habitual admitir conicidades con disminución de diámetro entre 6 y $13 \mathrm{~mm}$ por metro lineal de poste. Diferentes normas internacionales fijan unos intervalos o valores límite para dicha conicidad. Así, la normativa europea fija un intervalo de $6 \mathrm{~mm}$ a $16 \mathrm{~mm}$ por metro lineal (Aenor, 2011); la norma argentina fija una conicidad máxima de $10 \mathrm{~mm}$ por metro lineal (IRAM, 2007); y la norma ecuatoriana precisa un intervalo de entre 5 y $14 \mathrm{~mm}$ por metro lineal (INEN 1998).

En el presente estudio se han determinado las conicidades de los postes de eucalipto (Eucalyptus globulus Labill) y de pino amarillo del sur (Pinus sp.) de $8 \mathrm{~m}, 11 \mathrm{~m}$ y $12 \mathrm{~m}$ de clases 5,6 y 7, se han comparado ambas especies entre sí, y se han obtenido conicidades en torno a 6,0 $\mathrm{mm} / \mathrm{m}$ para el caso de los pinos amarillos del sur y en torno a $4,1 \mathrm{~mm} / \mathrm{m}$ para el caso del eucalipto.

\section{MATERIALES Y MÉTODOS}

Los postes preservados de eucalipto (Eucalyptus globulus Labill) fueron evaluados en las ciudades de Huancayo, Andahuaylas y Lima, mientras que los postes de pino amarillo del sur (Pinus sp.), procedentes del estado de Oregon, Estados Unidos de América, fueron evaluados en los almacenes de la empresa HGP Representaciones y Servicios EIRL, ubicados en la zona de Trapiche y Lurín, Lima.

Se estudiaron postes de eucalipto y de pino amarillo del sur preservados con sales hidrosolubles de $8 \mathrm{~m}$ - clase $7,11 \mathrm{~m}$ - clase 6 y $12 \mathrm{~m}$ - clases 5 y 6 . Para las medidas de longitud, se ha utilizado una cinta métrica de $25 \mathrm{~m}$ con $1 \mathrm{~cm}$ de división de escala y el diámetro se ha 
obtenido a partir de la circunferencia medida con una cinta métrica de $3 \mathrm{~m}$ con $1 \mathrm{~mm}$ de división de escala.

En cada uno de los postes de madera de cada especie y clase de este estudio, se midieron la longitud total, así como la circunferencia en la base y en la cabeza para obtener su conicidad.

Para el cálculo de la conicidad, se utilizó la fórmula señalada en la norma NTP 251.022 siguiente (Inacal, 2016):

$$
C_{o}(\mathrm{~mm} / \mathrm{m})=\frac{D_{p}-D_{c}}{L_{t}} \quad \begin{aligned}
& C_{o}: \operatorname{conicidad}(\mathrm{mm} / \mathrm{m}) \\
& D_{p}: \text { diámetro en el pie o base }(\mathrm{mm}) \\
& D_{c}: \text { diámetro en la cabeza }(\mathrm{mm}) \\
& L_{t}: \text { longitud tota } l(\mathrm{~m})
\end{aligned}
$$

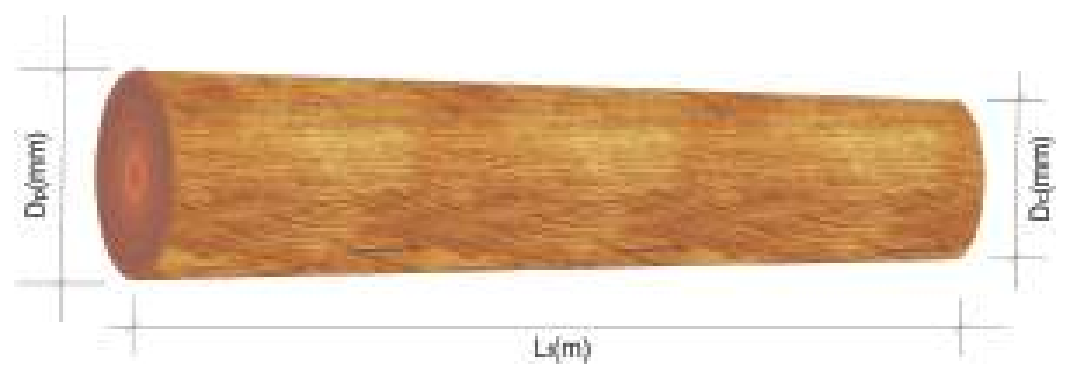

Figura 1. Mediciones para el cálculo de la conicidad de un poste Elaboración propia

Para el estudio de los datos, se efectuó un análisis de varianza (Anova) con un nivel de significancia del $5 \%$ para detectar las posibles diferencias significativas entre las dos especies, entre las distintas clases, y evaluar la influencia de la altura.

Previamente a la realización del análisis de varianza, se realizó un estudio de normalidad, independencia y homocedasticidad a partir de los datos de sesgo, curtosis y el Test de Levene.

Para todas las clases, se ha realizado un estudio de existencia de datos anómalos mediante el Test de Grubbs. Cada dato considerado estadísticamente como anómalo se ha examinado individualmente para evaluar su posible eliminación del conjunto total.

En los casos en los que se han detectado diferencias significativas entre los tratamientos, se ha realizado un análisis de intervalos de 
confianza a fin de estudiar entre cuáles de los tratamientos existen dichas diferencias. Dicho análisis se ha llevado a cabo mediante los test LSD de Fisher. El método LSD de Fisher es el método que proporciona más diferencias significativas: es una prueba muy eficiente para detectar diferencias verdaderas entre las medias.

Para efectuar los estudios estadísticos descriptivos, los análisis de varianza y los intervalos de confianza, se ha utilizado el programa Statgraphics Centurion XVI, Ver. 16.2.04.

\section{RESULTADOS Y DISCUSIÓN}

Los resultados de conicidad obtenidos por especie, altura y clase se exponen en la tabla 1.

Tabla 1

Resultados de conicidad

\begin{tabular}{|c|c|c|c|c|c|c|c|c|}
\hline \multirow{2}{*}{$\begin{array}{l}\frac{0}{0} \\
\Phi \\
\frac{0}{n} \\
\text { w }\end{array}$} & \multirow[b]{2}{*}{$\begin{array}{c}\text { Altura } \\
\text { (m) }\end{array}$} & \multirow[b]{2}{*}{ Clase } & \multicolumn{6}{|c|}{ Conicidad $(\mathrm{mm} / \mathrm{m})$} \\
\hline & & & $\begin{array}{l}\text { № de } \\
\text { datos }\end{array}$ & Media & $\begin{array}{l}\text { Desv. } \\
\text { típica }\end{array}$ & Rango & Sesgo & Curtosis \\
\hline \multirow{5}{*}{ 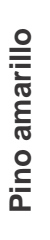 } & 8 & 7 & 347 & 5,68 & 1,27 & $(1,99-9,21)$ & 1,07 & 1,07 \\
\hline & 11 & 6 & 39 & 6,11 & 1,07 & $(4,63-8,12)$ & 0,75 & $-1,30$ \\
\hline & \multirow{2}{*}{12} & 5 & 103 & 6,51 & 1,20 & $(3,99-9,06)$ & 1,04 & 1,41 \\
\hline & & 6 & 153 & 6,45 & 0,98 & $(3,68-8,80)$ & $-1,92$ & 1,63 \\
\hline & \multicolumn{2}{|c|}{ Todos } & 642 & 6,03 & 1,23 & $(1,99-9,33)$ & 0,90 & 0,28 \\
\hline \multirow{5}{*}{ 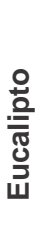 } & 8 & 7 & 550 & 4,19 & 1,24 & $(0,39-6,78)$ & $-0,93$ & $-0,52$ \\
\hline & 11 & 6 & 330 & 4,22 & 1,15 & $(0,87-6,94)$ & 0,25 & $-0,53$ \\
\hline & \multirow{2}{*}{12} & 5 & 215 & 4,21 & 1,11 & $(1,59-6,63)$ & 0,88 & $-1,50$ \\
\hline & & 6 & 495 & 4,03 & 1,07 & $(1,59-6,90)$ & 1,25 & $-0,18$ \\
\hline & \multicolumn{2}{|c|}{ Todos } & 1590 & 4,14 & 1,16 & $(0,39-6,94)$ & 0,54 & $-1,02$ \\
\hline
\end{tabular}

Elaboración propia

Todos los valores de sesgo y curtosis se encuentran dentro del intervalo $(-2,2)$ correspondiente a una distribución normal, por lo que no se puede rechazar la hipótesis de que los datos de cada una de las clases procedan de una distribución normal. 
En la tabla 2 se muestra el test de igualdad de varianzas para las dos especies.

Tabla 2

Test de varianzas

\begin{tabular}{cc}
\hline & $p$-valor \\
\hline Test de Levene & 0,08 \\
\hline
\end{tabular}

Elaboración propia

Puesto que el p-valor es superior a 0,05, se puede afirmar que no existen diferencias significativas entre las varianzas para un nivel de significación del 5\%.

Se han estudiado las series de datos, tanto por altura como por clase, y no se encontró ninguno que pudiera ser considerado como anómalo.

Se encontró además que todos los datos recogidos en la tabla 1 para el eucalipto y el pino amarillo del sur están dentro de las especificaciones de las diferentes normas actualmente utilizadas en el Perú. Así, los valores máximos de conicidad en el pino amarillo del sur se encuentran para postes de $12 \mathrm{~m}$ y las clases 5 y 6 , con valores de $6,51 \mathrm{~mm} / \mathrm{m}$ y $6,45 \mathrm{~mm} / \mathrm{m}$, inferiores a lo especificado por la norma ANSI 05.1 (ANSI, 2017), la cual admite valores máximos de $9,5 \mathrm{~mm} / \mathrm{m}$ y $9,6 \mathrm{~mm} / \mathrm{m}$, respectivamente. Lo mismo sucede con los valores máximos observados. El rango de conicidad del pino amarillo oscila entre $1,99 \mathrm{~mm} / \mathrm{m}$ y $9,33 \mathrm{~mm} / \mathrm{m}$, datos inferiores al los $9,5 \mathrm{~mm} / \mathrm{m}$ y $9,6 \mathrm{~mm} / \mathrm{m}$ permitidos por la norma.

Igual sucede en el caso del eucalipto, donde las medidas mayores de las conicidades se obtienen para la clase 6 en postes de $11 \mathrm{~m}$ y para la clase 5 en los de $12 \mathrm{~m}$. Estas conicidades, de $4,22 \mathrm{~mm} / \mathrm{m}$ y $4,21 \mathrm{~mm} / \mathrm{m}$ son inferiores a las especificadas por la norma NTP 251.022 (Inacal, 2017), que especifica unas conicidades máximas de $11,3 \mathrm{~mm} / \mathrm{m}$ y $10,9 \mathrm{~mm} / \mathrm{m}$. Lo mismo sucede si se evalúan los datos individuales: el rango de todas las conicidades del eucalipto está de $0,39 \mathrm{~mm} / \mathrm{m}$ a $6,94 \mathrm{~mm} / \mathrm{m}$, valores inferiores a los 11,3 mm/m y 10,9 mm/m máximos permitido por la norma.

Asimismo, los postes de eucalipto también están dentro de las especificaciones recogidas por la normativa argentina (IRAM, 2007), la cual fija un valor máximo de conicidad de $10 \mathrm{~mm} / \mathrm{m}$ para los postes de eucalipto. 
Los valores obtenidos se ubican dentro de los intervalos de conicidad obtenidos por Wood, Erickson y Dohr (1960) en sus estudios preliminares de las propiedades de resistencia de la madera para postes. Estos autores estudiaron las conicidades de diferentes especies utilizadas como postes de madera en Estados Unidos de América y obtuvieron, para el caso del pino amarillo, conicidades de $5,0 \mathrm{~mm} / \mathrm{m}$ para postes de $7,6 \mathrm{~m}\left(25^{\prime}\right), 6,2 \mathrm{~mm} / \mathrm{m}$ para postes de $9,1 \mathrm{~m}\left(30^{\prime}\right)$ y $9,4 \mathrm{~mm} / \mathrm{m}$ para

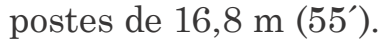

Además, los valores obtenidos para los postes de pino amarillo del sur son similares a los encontrados por otros autores para otras coníferas. Así, Martins y Dias (2012) obtuvieron una conicidad de $6,9 \mathrm{~mm} / \mathrm{m}$, y Morgado et al. (2009), una conicidad media de $6,3 \mathrm{~mm} / \mathrm{m}$, ambos para postes de $8 \mathrm{~m}$ de pino marítimo (Pinus sylvestris L.).

Igualmente, Wood et al. (1960), en el resto de las especies de su estudio, obtubieron valores similares. Así, para el abeto Douglas (Pseudotsuga menziesii (Mirb.) Franco), encontraron conicidades de $5,1 \mathrm{~mm} / \mathrm{m}$ para postes de $7,6 \mathrm{~m}, 3,8 \mathrm{~mm} / \mathrm{m}$ para postes de $9,1 \mathrm{~m}$ y $5,4 \mathrm{~mm} / \mathrm{m}$ para postes de 16,8 m. Para el alerce americano (Larix occidentalis Nutt.), obtuvieron conicidades de $5,8 \mathrm{~mm} / \mathrm{m}$ para postes de $7,6 \mathrm{~m}, 4,1 \mathrm{~mm} / \mathrm{m}$ para postes de $9,1 \mathrm{~m}$ y $7,1 \mathrm{~mm} / \mathrm{m}$ para postes de $16,8 \mathrm{~m}$. Para el pino contorta (Pinus contorta Douglas ex Loudon), obtuvieron conicidades de $7,2 \mathrm{~mm} / \mathrm{m}$ para postes de $7,6 \mathrm{~m}, 5,4 \mathrm{~mm} / \mathrm{m}$ para postes de $9,1 \mathrm{~m}$ y 9,8 $\mathrm{mm} / \mathrm{m}$ para postes de $16,8 \mathrm{~m}$. Finalmente, para el cedro rojo americano (Thuja plicata J. Don ex D. Don.), obtuvieron conicidades de $9,1 \mathrm{~mm} / \mathrm{m}$ para postes de $7,6 \mathrm{~m}, 7,7 \mathrm{~mm} / \mathrm{m}$ para postes de $9,1 \mathrm{~m} \mathrm{y} 13,4 \mathrm{~mm} / \mathrm{m}$ para postes de $16,8 \mathrm{~m}$.

Los valores obtenidos para el eucalipto están en consonancia con la bibliografía consultada, aunque ligeramente inferiores. Torrán et al. (2009) encontraron coeficientes de conicidad para postes de eucalipto (Eucalyptus grandis W.Hill ex Maiden) procedentes de Argentina entre $4,3 \mathrm{~mm} / \mathrm{m}$ y $7,0 \mathrm{~mm} / \mathrm{m}$ en el caso de postes de $8 \mathrm{~m}$ y entre $6,6 \mathrm{~mm} / \mathrm{m}$ y $8,8 \mathrm{~mm} / \mathrm{m}$ para postes de $12 \mathrm{~m}$. También son ligeramente inferiores a los obtenidos por Paula Silveira et al. (2011) en su estudio de postes de Eucalyptus camaldulensis Dehnh. en Pacaratu (Brasil) para el cual obtuvieron conicidades de $7,3 \mathrm{~mm} / \mathrm{m}$ en postes de $8 \mathrm{~m}, 7,9 \mathrm{~mm} / \mathrm{m}$ en postes de $11 \mathrm{~m}$ y $6,8 \mathrm{~mm} / \mathrm{m}$ en postes de $12 \mathrm{~m}$.

Se realizó un análisis de varianza para detectar si existen diferencias significativas tanto a nivel de especie como a nivel de clase; para 
ello, se compararon dentro de una misma especie los datos obtenidos para las distintas clases, así como los datos obtenidos en las diferentes especies para una misma clase y altura.

En las tablas 3 y 4 y las figuras 2 y 3 se muestran los resultados del análisis de diferencias significativas para la conicidad a nivel de especie.

Tabla 3

Análisis de varianza a nivel de especie

\begin{tabular}{lccccc}
\hline \multicolumn{1}{c}{ Fuente } & SS & g.l. & MS & F & p-Valor \\
\hline Altura & 1,02 & 1 & 1,02 & 0,73 & 0,39 \\
Clase & 8,60 & 2 & 4,30 & 3,08 & 0,04 \\
Especie & 1609,59 & 1 & 1609,59 & 1154,29 & $<0,01$ \\
Residuos & 3105,43 & 2227 & 1,39 & & \\
\hline Total & 4776,49 & 2231 & & & \\
\hline
\end{tabular}

Elaboración propia

Tabla 4

Intervalos de confianza LSD a nivel de especie

\begin{tabular}{lccc}
\hline Especie & $\begin{array}{c}\text { Límite inferior } \\
(\mathbf{m m} / \mathbf{m})\end{array}$ & $\begin{array}{c}\text { Límite superior } \\
(\mathbf{m m} / \mathbf{m})\end{array}$ & Grupo \\
\hline Pino amarillo & 5,96 & 6,09 & $\mathrm{~A}$ \\
Eucalipto & 4,11 & 4,18 & $\mathrm{~B}$ \\
\hline
\end{tabular}

Nota: Letras distintas en el grupo indican diferencias significativas para un nivel de confianza del $95 \%$.

Elaboración propia 


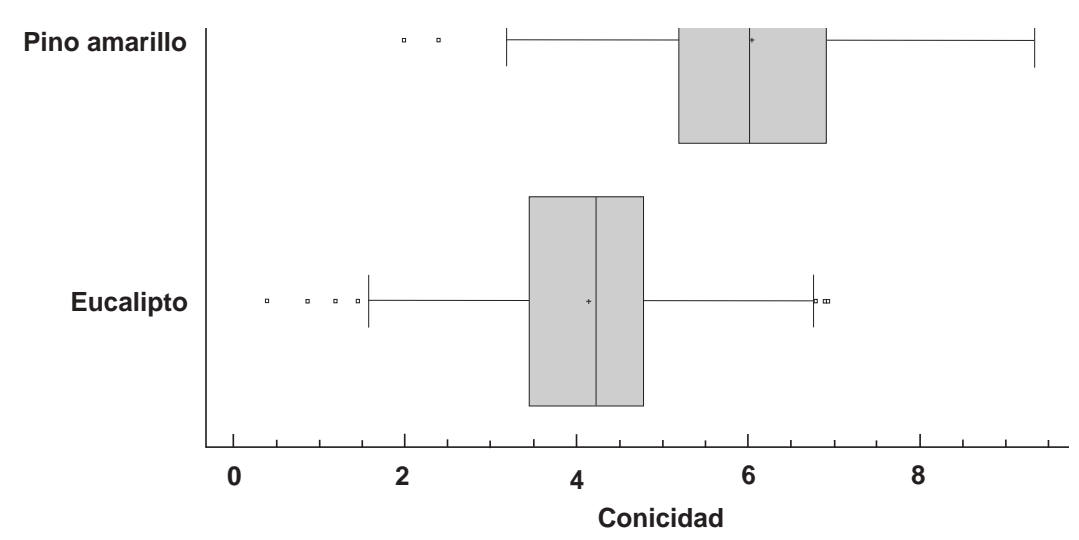

Figura 2. Diagrama de Box-Cox para las dos especies Elaboración propia

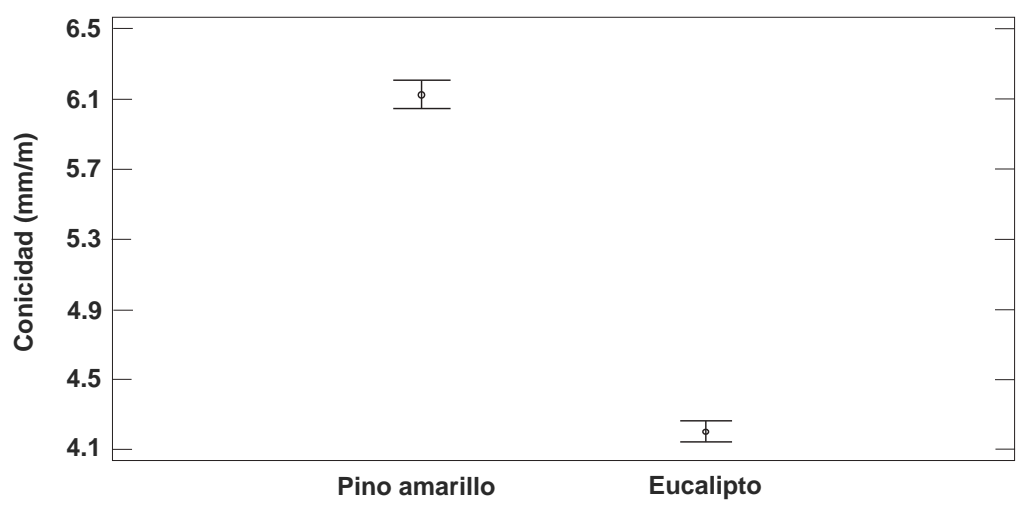

Figura 3. Diagrama de intervalos LSD para el pino amarillo Elaboración propia

Puesto que los p-valores correspondientes a la clase y la especie son inferiores a 0,05 , se puede afirmar que ambos factores influyen en la conicidad con un nivel de significancia de $5 \%$, y no sucede lo mismo para la altura del árbol, la cual presenta un $\mathrm{p}$-valor de 0,39 . Por lo tanto y de acuerdo con el estudio de los intervalos LSD, se puede afirmar que existe una diferencia significativa en la conicidad de ambas especies para un nivel de confianza del $95 \%$.

La existencia de estas diferencias entre especies está en consonancia con las propias especificaciones de cada especie recogidas por las 
normativas peruana y estadounidense. Así, la norma peruana NTP 251.022 (Inacal, 2017) especifica un rango de conicidades de $10,6 \mathrm{~mm} / \mathrm{m}$ a $11,3 \mathrm{~mm} / \mathrm{m}$ para el eucalipto, de acuerdo con su altura y clase, mientras que la norma estadounidense ANSI 05.1 (ANSI, 2017) especifica valores inferiores para los pinos amarillos del sur para fijarlos entre 8,8 $\mathrm{mm} / \mathrm{m}$ y $9,6 \mathrm{~mm} / \mathrm{m}$ de acuerdo con la altura y la clase.

En la tabla 5 y las figuras 4 al 8 se puede observar el análisis de diferencias significativas a nivel de clase, tanto intraespecie (figuras 4 y 5) como intraclase (figuras 6,7 y 8). Dicha tabla especifica los intervalos de confianza obtenidos mediante el análisis LSD para poder evaluar si esisten diferencias significativas entre los datos experimentales obtenidos para las diferentes clases en cuanto a conicidad se refiere. Es un análisis por clase y especie, y los intervalos de confianza indicados no deben confundirse con los rangos especificados en la tabla 1 a los cuales se les aplica la especificación de la norma NTP 251.022 (Inacal, 2017).

\begin{tabular}{lcccc}
$\begin{array}{l}\text { Tabla } 5 \\
\text { Intervalos de confianza a nivel de clase para las dos especies }\end{array}$ & \\
\hline Especie & Clase & $\begin{array}{c}\text { Límite inferior } \\
(\mathbf{m m} / \mathbf{m})\end{array}$ & $\begin{array}{c}\text { Límite superior } \\
(\mathbf{m m} / \mathbf{m})\end{array}$ & Grupo \\
\hline & 5 & 6,34 & 6,67 & A \\
Pino amarillo & 6 & 6,28 & 6,52 & A \\
& 7 & 5,59 & 5,77 & B \\
\hline & 5 & 4,10 & 4,31 & C \\
Eucalipto & 6 & 4,05 & 4,16 & C \\
& 7 & 4,12 & 4,25 & C \\
\hline
\end{tabular}

Nota: Letras distintas en el grupo indican diferencias significativas LSD para un nivel de confianza del $95 \%$

Elaboración propia 

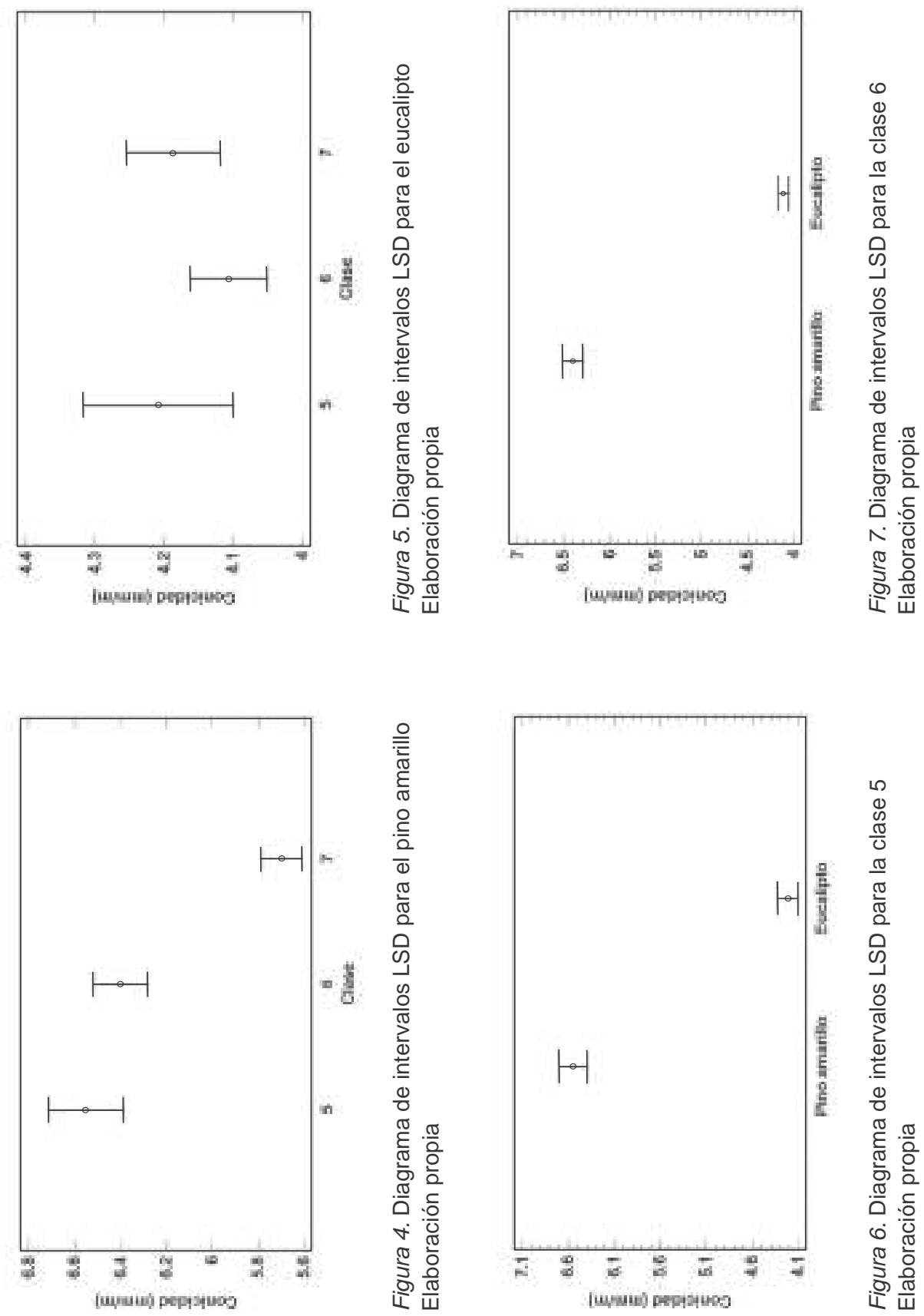

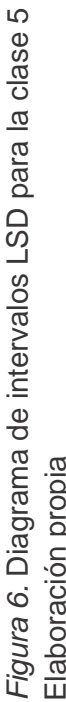




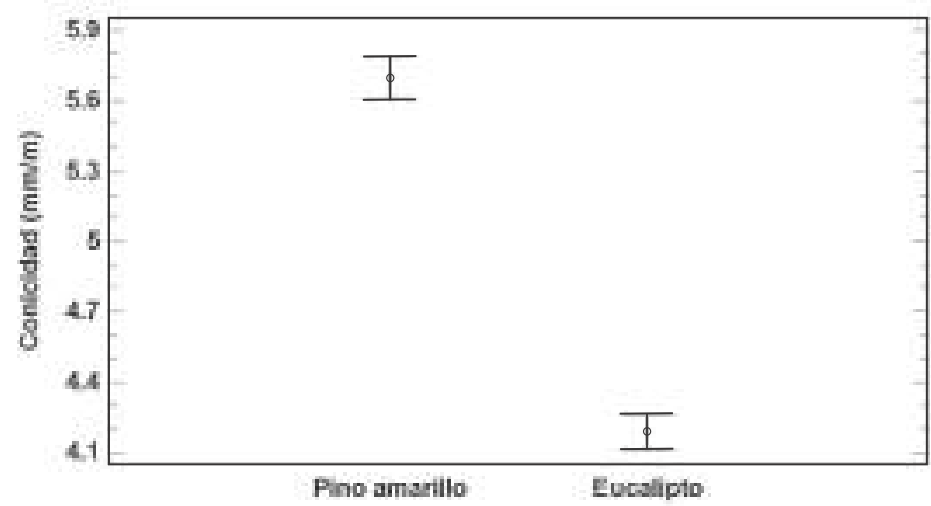

Figura 8. Diagrama de intervalos LSD para la clase 7 Elaboración propia

En el análisis intraespecies se observa, de acuerdo con los análisis de los intervalos de confianza LSD para un nivel de confianza del $95 \%$, que no existen diferencias significativas en la conicidad de las distintas clases en el eucalipto. Sin embargo, sí existen diferencias significativas en el pino amarillo, entre la clase 7 y el grupo formado por las clases 5 y 6 .

En el análisis intraclases se aprecia que, de acuerdo con los análisis de los intervalos de confianza LSD para un nivel de confianza del $95 \%$, existen diferencias significativas en la conicidad de ambas especies para una misma clase. Esto último corrobora los resultados obtenidos en el análisis global de las dos especies en los que se apreciaban diferencias significativas entre ambas. Puede comprobarse que esas diferencias significativas están presentes en cada una de las clases estudiadas.

\section{CONCLUSIONES}

- Las especies pino amarillo del sur (Pinus sp.) y eucalipto (Eucalyptus globulus Labill) estudiadas cumplen las especificaciones de las normas ANSI 05.1 y NTP 251.022.

- Existen diferencias significativas, para un nivel de confianza del 95 $\%$, entre la conicidad de las dos especies estudiadas.

- Para el caso del pino amarillo, no existen diferencias significativas, para un nivel de confianza del $95 \%$, entre las clases 5 y 6, pero sí con la clase 7 . 
- No existen diferencias significativas, para un nivel de confianza del $95 \%$, entre las diferentes clases para el caso del eucalipto.

\section{REFERENCIAS}

American National Standard Institute (ANSI). (2017). American National Standard for Wood Poles. Specifications and Dimensions. ANSI 05.1. Washington.

Asociación Española de Normalización y Certificación (AENOR). (2011). Madera estructural. Postes de madera para líneas aéreas. Norma UNE-EN 14229. Madrid.

González Pérez, H. (1988). La conicidad de los árboles y algunas posibles aplicaciones en el manejo de bosques. Revista de la Facultad Nacional de Agronomía - Medellín, 41(1), 51-60.

Instituto Argentino de Normalización y Certificación (IRAM). (2007). Postes y crucetas redondas de eucalipto preservados para líneas aéreas de energía eléctrica y sistemas de telecomunicaciones. Especificaciones. Norma IRAM 9513. Buenos Aires.

Instituto Ecuatoriano de Normalización (INEN). (1998). Postes de madera para líneas aéreas de energía eléctrica y telecomunicaciones. Requisitos. Norma NTE INEN 2 122. Quito.

Instituto Nacional de Defensa de la Competencia y la Propiedad Industrial (Indecopi). (2008). Postes de madera para líneas aéreas de conducción de energía. Glosario 2. ${ }^{\text {a }}$ edición. NTP 251.021. Lima.

Instituto Nacional de Defensa de la Competencia y la Propiedad Industrial (Indecopi). (1974 y revisada el 2012). Postes de madera para líneas aéreas de conducción de energía. Requisitos generales. 1. ${ }^{\text {a }}$ edición. NTP 251.022. Lima.

Martins, C. y Dias, A. 2012. Bending strength and stiffness of Portuguese Maritime Pine utility poles. Forest Products Journal, 62(2), 114-120.

Morgado, T. F. M., Rodriges, J., Machado, J. S., Dias, A. M. P. G. y Cruz, H. (2009). Bending and compression strength of Portuguesse Maritime pine small-diameter poles. Forest Products Journal, 59(4), 23-28. 
Paula Silveira, D., García Leite, H., Paula Silveira, V. y Nogueira Melido, R.C. (2011). Clasificação de árvores de eucalipto para postes em sistema agroforestal. Revista Árvore, 35(4), 875-882.

Torrán, E. A., Sosa Zitto, M. A., Cotrina A. D. y Piter, J. C. (2009). Bending strength and stiffness of poles of Argentinean Eucalyptus grandis. Maderas Ciencia y Tecnología, 11(1), 71-84.

Vignote, S., Martínez, I. y Villasante, A. (2013). La silvicultura y la calidad de madera. Lección inaugural 2013. Madrid: Universidad Politécnica de Madrid.

Wood, L. W., Erickson, E. C. O. y Dohr, A. W. (1960). Strength and related properties of wood poles. Philadelphia: ASTM Wood Pole Research Program. 